\title{
Isolated psoas hydatidosis mimicking ovarian neoplasm
}

\section{Upasana Baruah, Debabrata Barmon, Apoorva Tak*, Dimpy Begum}

Department of Gynaec-oncology, Dr. B Borooah Cancer Institute, A Unit of Tata Memorial Centre, Mumbai, Maharashtra, India

Received: 21 August 2021

Accepted: 13 September 2021

\author{
*Correspondence: \\ Dr. Apoorva Tak, \\ E-mail: drapoorvatak90@gmail.com
}

Copyright: (C) the author(s), publisher and licensee Medip Academy. This is an open-access article distributed under the terms of the Creative Commons Attribution Non-Commercial License, which permits unrestricted non-commercial use, distribution, and reproduction in any medium, provided the original work is properly cited.

\section{ABSTRACT}

We report a unique case of hydatid cyst in iliopsoas muscle in a 65-year-old postmenopausal female. She presented with lump abdomen. On performing exploratory laparotomy, we discovered iliopsoas hydatidosis. This case reports the diagnostic dilemmas that a hydatid cyst can pose and the implications it has on management.

Keywords: Psoas hydatidosis, Isolate muscle involved, Primary surgery, Albendazole

\section{INTRODUCTION}

Spectrum of manifestations in hydatid disease have been so diverse ranging from non-specific ones to anaphylaxis and death, that diagnosing it is often a dilemma. Humans are accidental intermediate host, of the larval stage of echinococcus granulosus, the causative parasite.

Echinococcal eggs are secreted from intestine of primary hosts mostly canines like dogs, which in turn are ingested and hatch in the intestine of intermediate host. Eggs as oncospheres disseminate via circulation, and get lodged in different organs, mostly the liver in $60-75 \%$ cases followed by the lung (15-25\%) cases.

Even in endemic regions, to find an isolated hydatid cyst of psoas muscle, is extremely rare.

In India majority cases have been reported from Andhra Pradesh and Tamil Nadu, constituting a prevalence of 10$15 \%$.

\section{CASE REPORT}

A P6L6 65-year-old postmenopausal female presented to our OPD with chief complaints of lump abdomen since 15 years and loss of appetite since 15 days. As elicited in history, the painless swelling had gradually increased in size over the years.

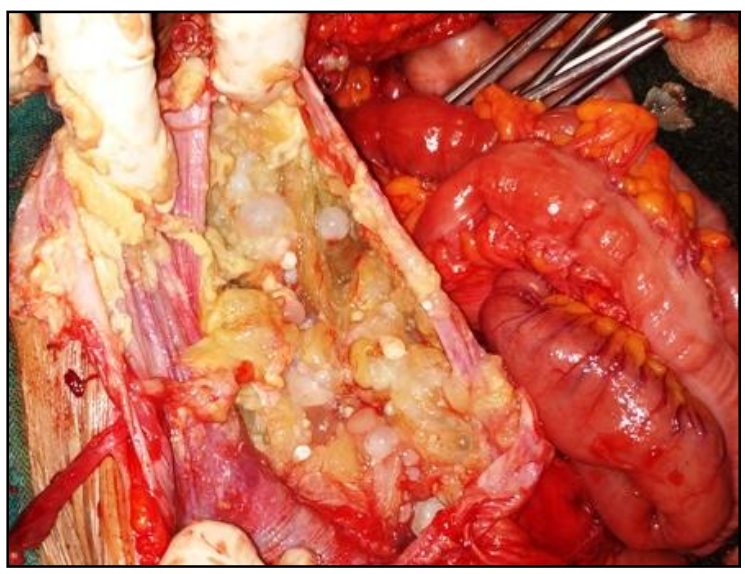

Figure 1: Intraoperative picture of PSOAS muscle hydatidosis.

\section{On clinical examination}

A 24-week abdominopelvic cystic mass was palpable, more towards the left side, with restricted mobility? Retroperitoneal in nature and lower pole could not be reached. On per speculum examination cervix and vagina 
were normal looking. PV and PR examination confirmed the above findings.

CT scan revealed a large multilocular solid cystic sol of about $13.5 \times 10.6 \times 24 \mathrm{~cm}$ on left side, uterus and right ovary were normal, mass abutted left distal ureter, left psoas, left lateral pelvic wall with compressed fat planes.

Among tumor markers, CA125 and CA19.9 were normal and cea was mildly raised. So upper GI endoscopy and colonoscopy were done and found to be normal. Patient was planned for exploratory laparotomy followed by frozen section.

Intraoperatively, uterus and $\mathrm{b} / \mathrm{l}$ tubes and ovaries were normal. Retroperitoneal cystic mass $12 \times 10 \mathrm{~cm}$ was seen arising from left psoas muscle and adherent to iliac bone and extending into left inguinal canal. The cystic mass ruptured during surgery as it was densely adherent to the pelvis. TAH+BSO+ complete excision of hydatid cyst from ilio-psoas muscle by retroperitoneal approach was done.

Gross specimen measured about $13 \times 13 \mathrm{~cm}$ with multiple fluid filled pearl white vesicles. Histological examination confirmed fetures of hydatid cyst with ectocyst and endocyst.

Post-operative period was uneventful, and patient was kept on injectable antibiotics for 5 days. Patient was discharged on 5th day with tablet albendazole for 24 days. Follow up period was uneventful. And no recurrence till date has been reported.

\section{DISCUSSION}

Echinococcosis is endemic in several countries of the world, including East Europe, South America and Mediterranean area. ${ }^{3}$

In humans, who act as intermediate host, hydatid cyst is formed by development of the larval stage of the parasite mostly in the liver and lungs. In certain cases, the embryo escapes the pulmonary circulation and enters the systemic circulation or systemic infection can occur due to rupture of hepatic cyst. ${ }^{4}$

Surgery is the mainstay of treatment in hydatid cyst and one should be careful to reduce the risk of possible intraoperative iatrogenic cyst rupture. Post-operative albendazole therapy reduces risk of recurrences. After a successful surgical operation, there is a recurrence rate of $2 \%$, while the survival rate is up to $95 \% .^{6}$
Hydatid cysts typically have an indolent course. Ultrasound is the gold standard method (sensitivity 93\%), however CT has a higher sensitivity for the diagnosis of peritoneal disease (up to $100 \%$ ). Computed tomography can diagnose daughter cysts and calcifications of the cyst wall.

Serological tests may also be useful for an accurate diagnosis. Moreover, IGg against the parasite antigen with ELISA (sensitivity 95\%) and indirect hemagglutination test may also be used for the diagnosis (sensitivity $87.5 \%$ ).

Intraoperative picture of hydatd cyst arising from psoas muscle.

\section{CONCLUSION}

Muscular hydatidosis mostly occurs as secondary localization of the disease, but the case we report here is very unique owing to the isolated involvement of psoas muscle. Echinococcosis can appear at any site in the human body, and so should always be considered in the differential diagnosis of cystic space-occupying lesions or unidentified tumour formations in patients from endemic areas.

\section{Funding: No funding sources Conflict of interest: None declared \\ Ethical approval: Not required}

\section{REFERENCES}

1. Arif SH, Wani NA, Zargar SA, Wani MA, Tabassum $\mathrm{R}$, Hussain $\mathrm{Z}$ et al. Albendazole as an adjuvant to the standard surgical management of hydatid cyst liver. Int J Surg. 2008;6:448-51.

2. Arora DR, Arora B. Echinococcusgranulosus. In medical parasitology. New Delhi, India: cbs publishers \& distributors. 2008;144-9.

3. Cattorini L, Trastulli S, Milani D, Cirocchi R, Giovannelli G, Avenia N et al. Ovarian hydatid cyst: a case report. Int J Surg Case Rep. 2011;2:100-2.

4. Dharsandia MV, Soni ST, Vegad MM. Ovarian hydatid cyst in pediatric patient commencing as ovarian tumor: a rare site of echinococcosis. Int J Prev Med. 2012;3:897-99.

5. Mushtaque M, Mir MF, Malik AA, Arif SH, Khanday SA, Dar RA. Atypical localizations of hydatid disease: experience from a single institute. Niger $\mathbf{J}$ Surg. 2012;18:2-7.

6. Sharma A, Sengupta P, Mandal S, Raychaudhuri G. Hydatid cyst of ovary mimicking ovarian neoplasm with its imprint cytology. Am J Case Rep. 2012;13:276-8.

Cite this article as: Baruah $\mathrm{U}$, Barmon $\mathrm{D}$, Tak $\mathrm{A}$, Begum D. Isolated psoas hydatidosis mimicking ovarian neoplasm. Int J Reprod Contracept Obstet Gynecol 2021;10:4317-8. 